\title{
Diferenças no crescimento e na composição corporal entre escolares de origem germânica e brasileira
}

\author{
Differences on the growth and body composition between school children of German and Brazilian origin
}

\author{
Everton Paulo Roman', Antonio de Azevedo Barros Filho
}

\section{RESUMO}

Objetivo: Analisar possíveis diferenças no crescimento e na composição corporal de crianças de cinco a dez anos de idade de origem étnica germânica e brasileira dos municípios de Marechal Cândido Rondon e de Foz do Iguaçu, Paraná.

Métodos: Estudo transversal, realizado nos anos de 2002 e 2003. Foram avaliadas 2.673 crianças, das quais 1.352 eram meninas, em 16 escolas públicas e particulares. Avaliou-se peso, estatura e as dobras cutâneas tricipital e subescapular. Obteve-se a medida derivada do índice de massa corpórea (IMC) e da soma das dobras tricipital e subescapular $(\mathrm{TR}+\mathrm{SE})$. Utilizou-se estatística descritiva e ANOVA para a comparação entre gênero e idade. $\mathrm{O}$ nível de significância foi $p<0,05$.

Resultados: Os escolares apresentaram valores de peso corporal e estatura próximos ao percentil 50, comparados com estudos de referência nacional e internacional. Quando comparados com os dados do Centers for Disease Control and Prevention (CDC), divulgados em 2000, eram obesos $10,9 \%$ dos escolares de etnia brasileira, 8,8\% dos meninos germânicos e 6,6\% das escolares germânicas. Nas dobras cutâneas, as meninas das duas etnias apresentaram valores superiores em relação aos meninos. Os valores de escore Z entre escola pública e particular apresentaram diferenças no peso corporal, estatura e IMC para as crianças germânicas. No entanto, quando analisadas crianças brasileiras e germânicas em escolas particulares, estas diferenças ocorreram apenas para o IMC.

Conclusões: Nas escolas públicas, as crianças germânicas são maiores quando comparadas às brasileiras; no entanto, nas escolas particulares, esta diferença desaparece, podendo sugerir que o ambiente exerce maior influência do que a origem étnica.

Palavras-chave: crescimento; composição corporal; grupos étnicos.

1Professor de Educação Física, mestre e doutorando em Saúde da Criança e do Adolescente na Faculdade de Ciências Médicas da Universidade Estadual de Campinas (FCM-Unicamp)

${ }^{2}$ Professor Associado do Departamento de Pediatria da FCM-Unicamp

\section{ABSTRACT}

Objective: Analyze possible differences on growth and body composition of children from five to ten years old of Germanic and Brazilian ethnic origin in the cities of Marechal Cândido Rondon and Foz do Iguaçu, Paraná, Brazil.

Methods: This cross-sectional study was carried out during 2002 and 2003 and enrolled 2,673 children (1,352 girls) in 16 public and private schools. Weight, height and triccipital (TR) and subscapular (SS) skinfolds were evaluated. The following variables were obtained: body mass index (BMI) and the sum of TR and SS. Descriptive statistic analysis was applied and the comparison of the variables between genders and among the ages strata was done by ANOVA, being significant $p<0,05$.

Results: The school children presented body weight and height close to the $50^{\text {th }}$ percentile, when these data were compared with national and international references. According to Centers for Disease Control and Prevention (CDC) reference curves, published in 2000 , obesity was present in $10.9 \%$ of the schoolchildren from Brazilian ethnic origin, in $8.8 \%$ of the boys and $6.6 \%$ of the girls of German ethnic background. The skin fold of the girls of both ethnicities presented higher values than the boys'. In public schools, Z scores showed that children of Germanic ethnicity presented higher body weight, height and BMI than those found in children of Brazilian ethnicity, but children of private schools showed difference only for BMI.

Conclusions: In the public schools, the children with Germanic background are bigger, but, in private schools, that difference disappears, suggesting that the environment exerts a stronger influence than ethnicity in reference to growth patterns.

Key-words: growth; body composition; ethnic groups.

Departamento de Pediatria da FCM-Unicamp

Caixa Postal 6111 - Barão Geraldo

CEP 13084-971 - Campinas/SP

E-mail: abarros@fcm.unicamp.br 


\section{Introdução}

O crescimento de crianças e adolescentes pode ser considerado como um dos mais importantes indicadores da qualidade de vida de um país ou como a extensão das distorções que possam existir em uma mesma população ${ }^{(1-}$

4). Recentes estudos realizados com crianças e adolescentes têm destacado a influência do contexto étnico e sociocultural como fator relevante para as discussões sobre as mudanças nos padrões de crescimento e de composição corporal $^{(5,6)}$.

Os termos "etnia" e "raça" têm significados diferentes, embora relacionados. "Raça" refere-se a um grupo biologicamente distinto, apresentando grande porcentagem de seus genes em comum por descendência, enquanto "etnia" referese a um grupo com características culturais específicas ${ }^{(7)}$.

O Brasil pode ser considerado um laboratório de etnias, pois abriga povos de praticamente todas as partes do mundo que migraram e constituíram aqui as suas famílias. Estudos realizados com nipo-brasileiros ${ }^{(8)}$ e árabes ${ }^{(9)}$ demonstram tendência de maior prevalência de sobrepeso e de obesidade em populações migrantes, podendo esta resultar do impacto da ocidentalização no estilo de vida e na dieta desses grupos étnicos.

A busca por informações sobre a inter-relação entre ambiente e hereditariedade em uma região ainda pouco explorada em relação a esta temática foi a motivação para realizar uma análise em relação ao crescimento, à composição corporal e aos índices de sobrepeso e de obesidade entre grupos étnicos, além de fazer algumas comparações com estudos nacionais $^{(4,10)} \mathrm{e}$ internacionais ${ }^{(11,12)}$.

O objetivo deste trabalho foi analisar possíveis diferenças no crescimento e na composição corporal entre escolares de cinco a dez anos de idade de origem étnica germânica e brasileira nas cidades de Marechal Cândido Rondon e Foz do Iguaçu, Paraná.

\section{Métodos}

Trata-se de um estudo transversal realizado nos anos de 2002 e 2003 em 16 escolas da rede pública e particular das cidades de Marechal Cândido Rondon e Foz do Iguaçu. Os escolares foram avaliados após aprovação do Comitê de Ética em Pesquisa da Faculdade de Ciências Médicas da Universidade Estadual de Campinas (FCM-Unicamp) e das Secretarias de Educação dos dois municípios. Antecedendo às avaliações, emitiu-se um termo de consen- timento livre e esclarecido, o qual foi entregue aos pais ou responsáveis pelas crianças, com o objetivo de obter autorização para o estudo e também para informar sobre os propósitos e os procedimentos técnicos da pesquisa, que estão de acordo com as normas que regulamentam pesquisas com seres humanos, segundo a Comissão Nacional de Saúde ${ }^{(13)}$.

A população deste estudo constituiu-se de todas as crianças de cinco a dez anos de idade matriculadas nas escolas públicas e particulares dos municípios de Marechal Cândido Rondon e Foz do Iguaçu durante o período da pesquisa.

A amostra foi constituída por 2.673 crianças de ascendência germânica ou brasileira. Consideraram-se como integrantes da pesquisa escolares que tivessem sobrenomes de pai e mãe de origem germânica (crianças germânicas) e escolares com sobrenomes de origem brasileira, espanhola e portuguesa (crianças brasileiras). Foram incluídas as crianças de ascendência germânica ou brasileira, que estavam dentro da faixa etária proposta e cujo pai ou responsável assinou o termo de consentimento livre e esclarecido.

Os dados foram coletados no horário de aula e, para a avaliação física, as crianças eram conduzidas até uma sala apropriada, determinando-se o peso corporal, a estatura e as dobras cutâneas tricipital e subescapular.

As medidas de peso e estatura foram realizadas de acordo com os procedimentos preconizados por Gordon et al ${ }^{(14)}$. O índice de massa corpórea (IMC) foi calculado pelo peso dividido pela estatura ao quadrado, sendo o peso expresso em quilogramas e a estatura em metros. O sobrepeso foi definido quando o IMC estava entre o percentil 85 e 95 e a obesidade quando o IMC era maior ou igual ao percentil 95, usandose como referência a curva do Centers for Disease Control and Prevention (CDC), divulgados em 2000 $0^{(11)}$.

As dobras cutâneas da região tricipital (TR) e subescapular (SE) foram avaliadas com um compasso da marca Lange, de acordo com os procedimentos descritos por Lohman et al $l^{(15)}$.

A composição corporal foi determinada pela soma das dobras $\sum(\mathrm{TR}+\mathrm{SE})$, de acordo com Frisancho ${ }^{(16)}$.

A análise dos dados foi descritiva. Para a comparação das variáveis em cada gênero e idade, foi utilizada a ANOVA. O nível de significância adotado foi $p<0,05$.

\section{Resultados}

Foram avaliadas 2.673 crianças, sendo 1.129 de origem germânica ( 585 meninos e 544 meninas) e 1.544 de origem brasileira (736 meninos e 808 meninas), que estudavam 
em escolas públicas e particulares das cidades de Marechal Cândido Rondon e Foz do Iguaçu, com idades entre cinco e dez anos, sendo considerada a idade decimal sugerida por Ross e Marfell-Jones ${ }^{(17)}$.

Para a estatura, meninos e meninas de etnia germânica apresentaram valores superiores dos seis aos dez anos de idade, quando comparados aos meninos e meninas de etnia brasileira (Tabela 1).

Para o IMC, os meninos de etnia germânica e brasileira aos dez anos de idade apresentaram valores superiores, quando comparados às meninas. Quanto aos aspectos étnicos, observaram-se valores superiores para os meninos de etnia brasileira aos seis anos de idade em relação aos de etnia germânica (Tabela 2).

Quando o IMC foi comparado com os resultados de outros estudos, os meninos da presente pesquisa apresentaram valores médios superiores em todas as faixas etárias, se comparados com os dados do $\mathrm{CDC}^{(11)}$ e com o estudo de Guedes \& Guedes ${ }^{(4)}$, conforme o Gráfico 1. Em relação às meninas, os valores foram superiores para as que pertenciam à etnia germânica e brasileira até os oito anos de idade, ocorrendo aproximação com os dados do $\mathrm{CDC}^{(11)}$ a partir dos nove anos (Gráfico 2).
A freqüência de crianças consideradas obesas ${ }^{(11)}$ foi, para os de etnia germânica, 8,8\% para os meninos (IC95\%=8,29,4 ) e $6,6 \%$ para as meninas (IC95\% $=6,1-7,1$ ). Observou-se também que $10,9 \%$ dos meninos e meninas de etnia brasileira eram obesos (IC95\%=10,2-11,7). Quanto à dobra cutânea tricipital, foram observadas diferenças dos cinco aos

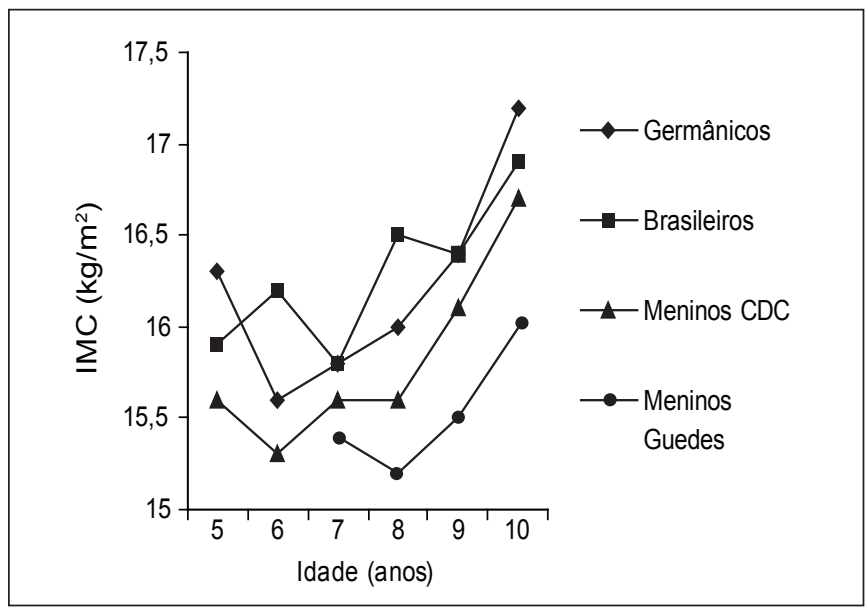

Gráfico 1 - Comparação dos resultados dos meninos de etnia germânica e brasileira no IMC com os estudos do $\mathrm{CDC}^{(11)}$ e Guedes \& Guedes $^{(4)}$

Tabela 1 - Valores de estatura em $\mathrm{cm}$ (média - $\bar{x}$, mediana - med e desvio padrão - dp) e número de crianças avaliadas de cinco a dez anos de idade, segundo gênero e etnia

\begin{tabular}{|c|c|c|c|c|c|c|c|c|c|c|c|c|c|c|c|c|}
\hline \multirow{3}{*}{ Idade } & \multicolumn{8}{|c|}{ Masculino } & \multicolumn{8}{|c|}{ Feminino } \\
\hline & \multicolumn{4}{|c|}{ Germânicos } & \multicolumn{4}{|c|}{ Brasileiros } & \multicolumn{4}{|c|}{ Germânicas } & \multicolumn{4}{|c|}{ Brasileiras } \\
\hline & $\mathbf{n}$ & $\overline{\mathbf{x}}$ & $d p$ & med & $\mathbf{n}$ & $\overline{\mathbf{x}}$ & $d p$ & med & $\mathbf{n}$ & $\overline{\mathbf{x}}$ & $d p$ & med & $\mathbf{n}$ & $\overline{\mathbf{x}}$ & $\mathrm{dp}$ & med \\
\hline 5 & 10 & 115,1 & 4,4 & 115,1 & 17 & 113,2 & 3,4 & 112,8 & 17 & 112,8 & 4,6 & 112,3 & 28 & 113,8 & 4,7 & 113,5 \\
\hline 6 & 111 & $119,0^{\dagger}$ & 5,5 & 119,4 & 99 & 117,2 & 5,3 & 117,1 & 89 & $118,5^{\dagger}$ & 5,7 & 118,0 & 96 & 116,8 & 5,2 & 116,8 \\
\hline 7 & 105 & $124,9^{\dagger}$ & 5,4 & 125,2 & 155 & 123,1 & 5,7 & 122,8 & 97 & $123,7^{\dagger}$ & 5,2 & 124,6 & 154 & 122,2 & 6,3 & 121,8 \\
\hline 8 & 129 & $130,0^{+}$ & 6,0 & 130,2 & 160 & 127,7 & 6,1 & 127,5 & 115 & $128,9^{\dagger}$ & 5,6 & 128,7 & 161 & 126,6 & 5,9 & 126,7 \\
\hline 9 & 102 & $134,8^{+}$ & 5,7 & 134,5 & 154 & 133,1 & 5,8 & 133,6 & 103 & $134,4^{\dagger}$ & 6,2 & 134,8 & 172 & 132,6 & 6,7 & 114,9 \\
\hline 10 & 128 & $140,8^{\dagger}$ & 7,3 & 140,2 & 151 & 138,4 & 6,7 & 138,6 & 123 & $139,2^{\dagger}$ & 8,2 & 138,9 & 197 & 138,2 & 6,7 & 138,1 \\
\hline
\end{tabular}

${ }^{\dagger}$ diferença estatisticamente significante em relação à etnia

Tabela 2 - Valores de índice de massa corpórea em $\mathrm{kg} / \mathrm{m}^{2}$ (média - $\bar{x}$, mediana - med e desvio padrão - dp) e número de crianças avaliadas de cinco a dez anos de idade, segundo gênero e etnia

\begin{tabular}{|c|c|c|c|c|c|c|c|c|c|c|c|c|c|c|c|c|}
\hline \multirow{3}{*}{ Idade } & \multicolumn{8}{|c|}{ Masculino } & \multicolumn{8}{|c|}{ Feminino } \\
\hline & \multicolumn{4}{|c|}{ Germânicos } & \multicolumn{4}{|c|}{ Brasileiros } & \multicolumn{4}{|c|}{ Germânicas } & \multicolumn{4}{|c|}{ Brasileiras } \\
\hline & $\mathbf{n}$ & $\overline{\mathbf{x}}$ & $d p$ & med & $\mathbf{n}$ & $\overline{\mathbf{x}}$ & $d p$ & med & $\mathbf{n}$ & $\overline{\mathbf{x}}$ & $d p$ & med & $\mathrm{n}$ & $\overline{\mathbf{x}}$ & $d p$ & med \\
\hline 5 & 10 & 16,8 & 2,7 & 16,3 & 17 & 16,8 & 2,7 & 15,9 & 17 & 16,8 & 2,4 & 16,5 & 28 & 17,2 & 2,1 & 17,0 \\
\hline 6 & 111 & 16,0 & 1,9 & 15,6 & 99 & $16,6^{\dagger}$ & 1,9 & 16,2 & 89 & 15,9 & 1,8 & 15,7 & 96 & 16,2 & 2,0 & 15,9 \\
\hline 7 & 105 & 16,4 & 2,0 & 15,8 & 155 & 16,5 & 2,3 & 15,8 & 97 & 16,0 & 2,3 & 15,7 & 154 & 16,5 & 2,4 & 15,8 \\
\hline 8 & 129 & 16,7 & 2,0 & 16,0 & 160 & 17,0 & 2,6 & 16,5 & 115 & 16,5 & 2,4 & 16,0 & 161 & 16,6 & 2,6 & 15,9 \\
\hline 9 & 102 & 16,8 & 2,2 & 16,4 & 154 & 17,0 & 2,5 & 16,4 & 103 & 16,7 & 2,6 & 16,2 & 172 & 16,8 & 2,7 & 16,0 \\
\hline 10 & 128 & $17,8^{*}$ & 3,0 & 17,2 & 151 & $17,8^{*}$ & 2,9 & 16,9 & 123 & 17,0 & 2,7 & 16,3 & 197 & 17,4 & 3,0 & 16,5 \\
\hline
\end{tabular}

"diferença estatisticamente significante em relação ao gênero; 'diferença estatisticamente significante em relação à etnia 
dez anos de idade, sendo maior nas meninas das duas etnias, se comparadas aos meninos. Em relação à dobra cutânea subescapular, notou-se diferenças dos seis aos dez anos de

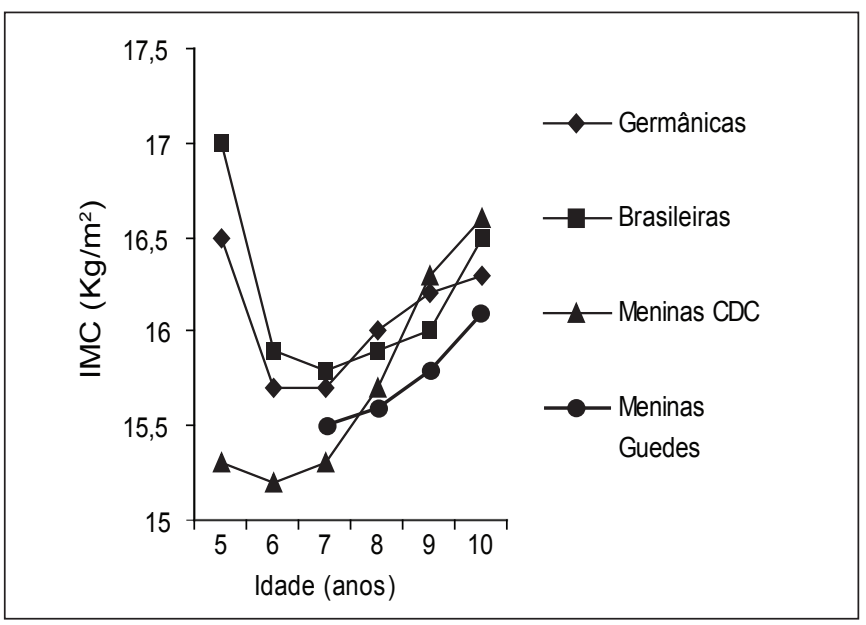

Gráfico 2 - Comparação dos resultados das meninas de etnia germânica e brasileira no IMC com os estudos do $\operatorname{CDC}^{(11)} \mathrm{e}$ Guedes e Guedes ${ }^{(4)}$ idade, sendo maior para as meninas de etnia germânica e brasileira, comparados aos meninos da mesma idade.

As medidas derivadas de soma das dobras $(\mathrm{TR}+\mathrm{SE})$ foram maiores na faixa de cinco a dez anos em meninas de etnia germânica e brasileira, comparadas aos meninos (Tabela 3).

Para os valores de escore $Z$ do peso e estatura, houve diferença estatisticamente significante entre as etnias na escola pública, onde, em média, crianças de etnia germânica apresentaram maiores valores no escore $\mathrm{Z}$ do que crianças de etnia brasileira. No entanto, quando consideradas as escolas particulares, estas diferenças não ocorreram (Tabela 4).

Não houve diferença estatisticamente significante no escore $\mathrm{Z}$ do IMC nos escolares de diferentes etnias que freqüentavam escolas públicas; porém, nas escolas particulares, em média, meninos e meninas de etnia brasileira apresentaram valores de escore $\mathrm{Z}$ maiores do que meninos e meninas de etnia germânica.

Tabela 3 - Valores de somatória das dobras cutâneas da região tricipital e subescapular $(\mathrm{mm})$ (média - $\overline{\mathrm{x}}$, mediana - med e desvio padrão - dp) e número de crianças avaliadas de cinco a dez anos de idade, segundo gênero e etnia

\begin{tabular}{|c|c|c|c|c|c|c|c|c|c|c|c|c|c|c|c|c|}
\hline \multirow{3}{*}{ Idade } & \multicolumn{8}{|c|}{ Masculino } & \multicolumn{8}{|c|}{ Feminino } \\
\hline & \multicolumn{4}{|c|}{ Germânicos } & \multicolumn{4}{|c|}{ Brasileiros } & \multicolumn{4}{|c|}{ Germânicos } & \multicolumn{4}{|c|}{ Brasileiros } \\
\hline & $\mathbf{n}$ & $\overline{\mathbf{x}}$ & $d p$ & med & $n$ & $\overline{\mathbf{x}}$ & $\mathrm{dp}$ & med & $\mathrm{n}$ & $\overline{\mathbf{x}}$ & $\mathrm{dp}$ & med & $\mathbf{n}$ & $\overline{\mathbf{x}}$ & $d p$ & med \\
\hline 5 & 10 & 21,2 & 9,9 & 18,3 & 17 & 22,3 & 12,2 & 19,0 & 17 & $24,7^{*}$ & 10,9 & 21,0 & 28 & $24,9^{*}$ & 8,5 & 24,0 \\
\hline 6 & 111 & 18,7 & 8,1 & 16,5 & 99 & 19,6 & 6,6 & 18,0 & 89 & $21,1^{*}$ & 7,1 & 19,0 & 96 & $22,2^{*}$ & 8,4 & 19,3 \\
\hline 7 & 105 & 20,1 & 8,5 & 18,0 & 155 & 20,3 & 9,2 & 17,5 & 97 & $22,7^{*}$ & 9,7 & 21,0 & 154 & $24,0^{*}$ & 10,2 & 21,0 \\
\hline 8 & 129 & 22,8 & 10,3 & 20,0 & 160 & 22,8 & 10,8 & 19,5 & 115 & $25,4^{*}$ & 10,2 & 23,0 & 161 & $25,9^{*}$ & 12,4 & 22,0 \\
\hline 9 & 102 & 22,2 & 10,4 & 19,8 & 154 & 22,4 & 10,0 & 20,0 & 103 & $27,0^{*}$ & 12,4 & 23,0 & 172 & $26,6^{*}$ & 12,7 & 22,3 \\
\hline 10 & 128 & 26,7 & 13,7 & 22,0 & 151 & 26,7 & 13,4 & 23,0 & 123 & $28,9^{*}$ & 12,8 & 25,5 & 197 & $29,3^{*}$ & 13,2 & 25,5 \\
\hline
\end{tabular}

*diferença estatisticamente significante em relação ao gênero

Tabela 4 - Valores de escore Z para peso, índice de massa corpórea e estatura segundo tipo de escola

\begin{tabular}{|c|c|c|c|c|c|c|c|c|c|c|c|c|}
\hline & \multicolumn{6}{|c|}{ Germânicos - escolas públicas } & \multicolumn{6}{|c|}{ Brasileiros - escolas públicas } \\
\hline & \multicolumn{3}{|c|}{ Masculino } & \multicolumn{3}{|c|}{ Feminino } & \multicolumn{3}{|c|}{ Masculino } & \multicolumn{3}{|c|}{ Feminino } \\
\hline & $\mathbf{n}$ & $\overline{\mathbf{x}}$ & $d p$ & $\mathbf{n}$ & $\overline{\mathbf{x}}$ & $d p$ & $n$ & $\overline{\mathbf{x}}$ & $d p$ & $\mathbf{n}$ & $\overline{\mathbf{x}}$ & $d p$ \\
\hline Peso Z & 470 & $0,33^{*}$ & 1,08 & 426 & $0,12^{\ddagger}$ & 1,06 & 581 & 0,14 & 1,07 & 640 & $-0,01$ & 1,09 \\
\hline IMC Z & 470 & 0,15 & 1,17 & 426 & 0,02 & 1,04 & 581 & 0,21 & 1,04 & 640 & 0,07 & 1,06 \\
\hline \multirow[t]{4}{*}{ Est. Z } & 470 & $0,39^{*}$ & 1,06 & 426 & $0,27^{\ddagger}$ & 1,08 & 581 & $-0,02$ & 1,04 & 640 & $-0,06$ & 1,06 \\
\hline & \multicolumn{6}{|c|}{ Germânicos - escolas particulares } & \multicolumn{6}{|c|}{ Brasileiros - escolas particulares } \\
\hline & \multicolumn{3}{|c|}{ Masculino } & \multicolumn{3}{|c|}{ Feminino } & \multicolumn{3}{|c|}{ Masculino } & \multicolumn{3}{|c|}{ Feminino } \\
\hline & $\mathbf{n}$ & $\overline{\mathbf{x}}$ & $d p$ & $\mathbf{n}$ & $\overline{\mathbf{x}}$ & dp & $\mathbf{n}$ & $\overline{\mathbf{x}}$ & dp & $\mathbf{n}$ & $\overline{\mathbf{x}}$ & $\mathrm{dp}$ \\
\hline Peso Z & 115 & 0,66 & 0,98 & 118 & 0,36 & 1,04 & 155 & 0,71 & 1,00 & 168 & 0,47 & 1,06 \\
\hline IMC Z & 115 & 0,43 & 1,00 & 118 & 0,08 & 1,32 & 155 & $0,67^{*}$ & 0,99 & 168 & $0,39 \ddagger$ & 1,09 \\
\hline Est. Z & 115 & 0,67 & 1,00 & 118 & 0,55 & 0,96 & 155 & 0,45 & 0,96 & 168 & 0,47 & 1,07 \\
\hline
\end{tabular}

"diferença estatisticamente significativa entre etnias para o gênero masculino; ‡diferença estatisticamente significativa entre etnias para o gênero feminino 


\section{Discussão}

Os resultados obtidos neste estudo indicam que, nas escolas públicas, as crianças germânicas são maiores quando comparadas às brasileiras; no entanto, nas escolas particulares, esta diferença desaparece. Os achados mostram maior prevalência de sobrepeso e obesidade em escolares de etnia brasileira, quando comparados aos de etnia germânica. As crianças das duas etnias avaliadas apresentaram valores de peso e estatura próximos ao percentil 50 do $\mathrm{CDC}^{(11)}$ e do estudo de Marcondes $^{(10)}$.

Em relação ao IMC, quando os resultados foram comparados aos do $\mathrm{CDC}^{(11)} \mathrm{e}$ ao estudo regional realizado na cidade de Londrina por Guedes \& Guedes ${ }^{(4)}$, os meninos pertencentes à etnia germânica e brasileira apresentaram valores na mediana superiores em todas as faixas etárias avaliadas, enquanto as meninas até os oito anos de idade mostraram valores superiores, porém, a partir dos nove e dez anos houve uma tendência de aproximação nos resultados. Observou-se também que 10,9\% das crianças de etnia brasileira apresentaram valor maior ou igual ao percentil 95 , comparado ao referencial do $\mathrm{CDC}^{(11)}$.

Nas dobras cutâneas tricipital e subescapular e na soma das dobras $(\mathrm{TR}+\mathrm{SE})$, as meninas deste estudo apresentaram valores superiores em todas as faixas etárias, comparadas a estudos de referência internacional, conforme $\operatorname{CDC}^{(11)} \mathrm{e}$ Frisancho $^{(12)}$.

As cidades de Marechal Cândido Rondon e Foz do Iguaçu, apesar de geograficamente próximas, apresentam costumes e características totalmente diferentes. A primeira teve sua colonização realizada na década de 1950, durante o pós-guerra, por alemães provenientes da Alemanha e do Estado de Santa Catarina, sendo considerada atualmente a cidade mais germânica do Paraná. A segunda, localizada na fronteira entre o Brasil, Argentina e Paraguai, tem sua economia baseada principalmente no turismo e é conhecida como uma cidade que abriga povos de diversos países.

Com as diferenças étnicas, culturais e econômicas claramente evidenciadas entre as duas cidades, investigou-se a possibilidade de existirem diferenças no crescimento e na composição corporal das crianças dessas etnias nas duas cidades, pois, embora pertencentes à mesma região, a caracterização étnica poderia ser um fator de influência.

Inicialmente foram encontradas algumas dificuldades para a realização desta pesquisa nos anos de 2002 e 2003, mas, após a realização de reuniões com os pais e diretores das escolas para explicar os objetivos e os métodos do estudo, o trabalho pôde ser realizado dentro das expectativas estabelecidas pelos pesquisadores, referentes à aleatoriedade e ao número de pessoas avaliadas.

Quanto à estatura, os meninos de etnia germânica e brasileira são mais altos, quando comparados às meninas. Os valores da estatura estão próximos aos valores sugeridos por Tanner ${ }^{(18)}$ de um ganho estatural em média de $5 \mathrm{~cm}$ por ano.

As transformações ocorridas na sociedade brasileira nas últimas décadas, como a melhora na qualidade de vida e saúde e a erradicação de doenças, podem ter reflexo direto na estatura, a qual é considerada um dos fatores que melhor reflete o histórico nutricional do indivíduo.

Foi observado, pelos valores apresentados em escore Z, que crianças germânicas e brasileiras de escolas públicas apresentam estatura menor quando comparadas a crianças germânicas e brasileiras que estudam em escolas particulares; mesmo assim, os valores da mediana estão próximos aos de Marcondes $^{(10)}$ e aos dados do CDC $^{(11)}$.

Os resultados do IMC foram semelhantes entre meninos e meninas; porém, quando comparados ao estudo regional de Guedes \& Guedes ${ }^{(4)}$ e aos dados do $\mathrm{CDC}^{(11)}$, os valores foram maiores nos escolares deste estudo. Ainda em relação ao IMC, entre as crianças das escolas particulares, os valores de escore $\mathrm{Z}$ das crianças brasileiras foram maiores que os das germânicas, que indica um maior percentual de crianças obesas.

O IMC pode ser considerado como um critério importante para a detecção de sobrepeso e obesidade não somente na fase adulta, mas também na infância. Diversos estudos mostram tendência de aumento do IMC em todas as faixas etárias em diferentes níveis socioeconômicos. A transição nutricional que tem atingido diversas populações, fundamentalmente em países em amplo processo de desenvolvimento como o Brasil, pode ser considerada como um dos fatores relevantes para tais resultados.

A prevalência de obesidade, principalmente entre crianças de origem étnica brasileira, pode estar associada possivelmente ao seu estilo de vida e aos níveis de atividade física. Nesta linha de raciocínio, Malina e Bouchard ${ }^{(7)}$ relataram que os fatores socioculturais têm parcela significativa no desenvolvimento da obesidade.

Estudos realizados em diversos países relatam o aumento nos índices de sobrepeso e obesidade em diferentes grupos sociais e, principalmente, em diferentes etnias ${ }^{(11,19)}$. Monteiro et $a^{(20)}$ mostram aumento da prevalência da obesidade no Brasil, com distribuição em todos os estratos econômicos da população. 
Em relação às dobras cutâneas, as meninas de origem germânica e brasileira apresentaram valores superiores, comparados aos meninos, praticamente em todas as faixas etárias avaliadas, o que também já havia sido relatado na literatura ${ }^{(21)}$.

Quando se analisaram os dados por meio de escore Z, os resultados mais importantes foram detectados em relação à etnia, mostrando que crianças germânicas de escola pública apresentaram diferenças no peso corporal e na estatura, comparadas às crianças de etnia brasileira; entretanto, quando comparadas às crianças de escolas particulares, estas diferenças não foram evidenciadas.

Nas escolas particulares, foi observada diferença estatisticamente significante no escore $\mathrm{Z}$ do IMC, com maior valor médio em meninos e meninas de etnia brasileira, comparado ao observado nas crianças de etnia germânica, não havendo diferenças no IMC entre as duas etnias na escola pública.

Cabe ressaltar que este é um dos poucos trabalhos sobre características étnicas de crianças brasileiras e poderá contribuir para comparações entre amostras e populações semelhantes em outras regiões do país.

O Brasil é um país colonizado por diversas etnias e, assim, é um local propício para realizar novos estudos com diferentes grupos étnicos em todas as suas regiões, de forma a

\section{Referências bibliográficas}

1. Goldstein $\mathrm{H}$, Tanner JM. Ecological considerations in the criterion and the use of child growth standards. Lancet 1980;1:582-5.

2. Keller W. Epidemologia del atraso del crecimiento. In: Waterlow JC, editor. Retraso del crecimiento linear en los paises en vias de desarrollo. Genebra: Nestlé Nutrition; 1987. p. 9-12.

3. Gopalan C. Stunting: significance and implication for public health policy. In: Waterlow JC, editor. Linear growth retardation in less developed countries. New York: Raven Press; 1988. p. 265-84.

4. Guedes DP, Guedes JE. Crescimento, composição corporal e desempenho motor de crianças e adolescentes. São Paulo: CLR Balieiro, 1997.

5. Castilho LV, Lahr MM. Secular trends in growth among urban Brazilian children of European descent. Ann Hum Biol 2001;28:564-74.

6. Balaban G, Silva GA. Prevalência de sobrepeso e obesidade em crianças e adolescentes de uma escola da rede privada de Recife. J Pediatr (Rio J) 2001;77:96-100.

7. Malina RM, Bouchard C. Growth, maturation, and physical activity. $1^{\text {st }}$ ed. Champaign: Human Kinetics; 1991.

8. Taniguchi C, Gimeno SG, Ferreira, SR, Japanese-Brazilian Diabetes Study Group. Características antropométricas de nipo-brasileiros. Rev Bras Epidemiol 2004; 7:423-33.

9. Roman EP, Barros Filho AA. Prevalência de sobrepeso e obesidade em escolares de etnia árabe na cidade de Foz do Iguaçu. Rev Paul Pediatr 2004;22:23-30.

10. Marcondes E. Normas para o diagnóstico e classificação dos distúrbios de crescimento e da nutrição. Pediatr (SP) 1982;4:307-28.

11. Kuczmarski RJ, Ogden CL, Guo SS, Grummer-Strawn LM, Flegal KM, Mei Z et al. 2000 CDC Growth Charts for the United States: methods and development. Vital Health Stat 2002;11:1-190. monitorar e melhor interpretar os fatores que possivelmente influenciam e determinam o crescimento e a composição corporal nestas faixas etárias.

Entretanto, é importante destacar alguns fatores que não foram avaliados neste estudo: a ingestão calórica diária de alimentos, o peso e a estatura dos pais, as horas diárias de sono, a ocupação do tempo livre e o nível de atividade física. Tais fatores poderiam ser melhor avaliados com a realização de novos estudos.

O peso corporal e a estatura das crianças de ambos os gêneros e etnias aqui estudadas foram próximos do percentil 50 de referência nacional e internacional. As meninas apresentaram valores superiores aos dos meninos nas dobras cutâneas tricipital e subescapular e, conseqüentemente, na soma das dobras, situando-se acima do percentil 50, comparadas aos padrões de referência internacional.

$A$ análise dos valores de escore $Z$ de peso e estatura em ambos os gêneros, independente da idade das crianças avaliadas, mostraram que, em escolas particulares, a etnia não interferiu nessas medidas. Entretanto, em escolas públicas, as características étnicas acabam se manifestando, sugerindo que o meio ambiente no qual as crianças estão inseridas exerce maior influência em relação às variáveis de crescimento e composição corporal do que as próprias características étnicas.

12. Frisancho AR. Anthropometric standards for the assessment of growth and nutritional status. Michigan: The University of Michigan Press; 1990.

13. Ministério da Saúde. Conselho Nacional de Saúde. Resolução no. 196 de 10/10/96 sobre pesquisa envolvendo seres humanos. DOU 1996 Out 16; no. 201, seção 1:21082-210-85.

14. Gordon CC, Chumlea WC, Roche AF. Stature, recumbent length and weight In: Lohman TG, Roche AF, Martorell R, editors. Anthropometric standardization reference manual. Champain: Human Kinetics Books; 1988. p. 3-8.

15. Lohman TG, Roche AF, Martorell R. Anthropometric standardization reference manual. Champaign: Human Kinetics Books; 1988.

16. Frisancho AR. New norms of upper limb fat and muscle areas for assessment of nutritional status. Am J Clin Nutr 1981;34:2540-5.

17. Ross WD, Marfell-Jones MJ. Kinanthropometry. In: MacDougali HA, Wernger $\mathrm{H}$, Green $\mathrm{HJ}$, editors. Physiological testing of the elite Canadian association of sport science. Otawa: Mutual; 1982. p. 223-308.

18. Tanner, JM. A history of the study of human growth. Cambridge: University Press; 1981.

19. Saxena S, Ambler G, Cole TJ, Majeed A. Ethnic group differences in overweight and obese children and young people in England: cross sectional survey. Arch Dis Child 2004;89:30-6.

20. Monteiro CA, Lenise M, Souza ALM, Popkin BM. Da desnutrição para a obesidade: a transição nutricional no Brasil. In: Monteiro CA, editor. Velhos e novos males da saúde no Brasil: a evolução do Brasil e suas doenças. São Paulo: Hucitec; 2000. p. 247-55.

21. Frisancho AR. Role of calorie and protein reserves on human growth during childhood and adolescent in a mestizo Peruvian population. In: Greene L, Johnston FE, editors. Social and biological predictors of nutrition status, physical growth and neurological development. New York: Academia Press; 1980. p. 49-57. 